\title{
Wavelet Order Statistics Filters for Image Denoising
}

\author{
F. Gallegos-Funes, J. Martínez-Valdés, R. Cruz-Santiago, J. López-Bonilla \\ National Polytechnic Institute of Mexico, Mechanical and Electrical Engineering Higher School \\ Av. IPN s/n, ESIME SEPI Electrónica, Lindavista, 07738, Mexico City
}

Received 5 December 2008, accepted in final revised form 15 April 2009

\begin{abstract}
This paper presents the wavelet order statistics filters for the removal of impulsive and speckle noise in color image applications. The proposed filtering scheme is defined as two filters in the wavelet domain to conform to the structure of a general filter that can be modified in some headings. The first filter is based on redundancy of approaches and the second one is the wavelet domain iterative center weighted median algorithm. With the structure of the proposed filter different implementations for the estimation of the noisy sample are carried out using different order statistics algorithms that by their good performance can be beneficial in color image processing applications.
\end{abstract}

Keywords: Wavelet domain; Order statistics filters.

(C) 2009 JSR Publications. ISSN: 2070-0237 (Print); 2070-0245 (Online). All rights reserved.

DOI: 10.3329/jsr.v1i2.2311

\section{Introduction}

Many different classes of filters have been proposed for removing noise from images [13]. They are classified into several categories depending on specific applications. The order statistics filters are designed to suppress noise of different nature, they can remove impulsive noise and guarantee detail preservation [1-3]. On the other hand, the filters based in the wavelet domain provide a better performance in terms of noise suppression in comparison with different space domain filters [4, 5].

In this paper we propose a filter that works in the wavelet domain and uses different order statistics algorithms in its filtering scheme. The proposed filter is composed by two filters that carry out the impulsive noise suppression in the wavelet domain. The first filter based on redundancy of approaches [6] smoothes the low frequencies (LF) of the noisy image by means of a double convolution operation (first of decomposition and after reconstruction) between the wavelet coefficients and the samples of the corrupted image. The second one is the wavelet domain iterative center weighted median filter [7] that provides an analysis of the histograms of the wavelet coefficients of several pairs of images (original and corrupted) through different scales and carries out an improved estimation of the variance field of the noisy wavelet coefficients of the image. With the

\footnotetext{
*Corresponding author: fgallegosf@ipn.mx
} 
aid of the estimator of minimum mean square error this second filter finally obtains the filtered wavelet coefficients of the approaches and details of the image.

The structure of the proposed filter is designed so that it can be modified in the sections of the first and second detection of the noisy sample in addition to the estimation algorithm, all contained in the wavelet domain iterative center weighted median filter [7] to increase its robustness and to improve its performance in the task of impulsive noise suppression. We introduce in the proposed filter the algorithms of the SD-ROM, adaptive center weighted median, FIR median hybrid, and median M-type KNN Filters [1, 2, 8, 9], and adapt them to work in the wavelet domain into the two detection blocks proposed in this paper. Simulation results have demonstrated that the proposed filters can outperform other traditional wavelet color image filters at least for high values of noise contamination by balancing the tradeoff between noise suppression, fine detail preservation, and color retention.

\section{Wavelet Domain Order Statistics Filtering Scheme}

The proposed filter constitute two filters: the filter based on redundancy of approaches [6] and the wavelet domain iterative center weighted median filter (ICWMF) [7] as shown in Fig. 1. For each color component of the noisy image it is necessary to apply all the steps contained in this structure. This technique applies up to 5 scaling levels for the details and only 1 scaling level for the approaches. Other operations are indicated to make clearer the wavelet analysis that it is carried out in this paper. We modify this structure in the block of the ICWMF. For that reason, the expressions used by the ICWMF to calculate the improved estimation of the variance field of the noisy wavelet coefficients will be required to indicate when and where different proposed filtering algorithms will take place to improve the performance of the proposed filter.

The first stage of the ICWMF [7] that detects if a sample contains noise or not is given by:

$$
\hat{\sigma}_{g s}^{2}(k)= \begin{cases}\widetilde{\sigma}_{g s}^{2}(k), & \text { if } \lambda_{s} \geq \lambda_{t h}, \\ \operatorname{med}_{c w}^{i}\left(\widetilde{\sigma}_{g s}^{2}(j)\right), & \text { otherwise }\end{cases}
$$

where $\tilde{\sigma}_{g s}^{2}$ is the variance field estimated previously, $k$ is central sample in the filter window, $j$ is one of the $N$ sample contained into the window, $\lambda_{s}$ is the standard deviation of the preliminary estimate of the signal coefficients variances $\tilde{\sigma}_{s}^{2}(k)$ in each scale, $\lambda_{\text {th }}=\sum_{s} \lambda_{s} 2^{-s} / \sum_{s} 2^{-s}$ is the discriminating threshold, $s$ is the scale used in the wavelet analysis, and $2^{-s}$ is the weighting function [7]

The SD-ROM, adaptive center weighted median, and median M-type KNN [1, 8, 9] algorithms were applied to the proposed filter as a first detection block. But the FIR Median Hybrid algorithm [2] was applied as a second detection block because this algorithm only constitutes the part of estimation of the noisy sample value (only if the sample was detected of this way) and the proposed filter can continue operating in all its 
sections in the same way. For this reason it is necessary to present the expression for the second detection block contained in the proposed filter structure [7]:

$$
\operatorname{med}_{c w}^{i}\left(\tilde{\sigma}_{g s}^{2}(j)\right)= \begin{cases}\operatorname{med}\left(\tilde{\sigma}_{g s}^{2}(j)\right) & \text { if } \quad \tilde{\sigma}_{g s}^{2}(k) \leq \gamma \sigma_{n}^{2} \\ \tilde{\sigma}_{g s}^{2}(k)+\operatorname{med}\left(\tilde{\sigma}_{g s}^{2}(j)-\tilde{\sigma}_{g s}^{2}(k)\right) & \text { otherwise }\end{cases}
$$

The proposed filter uses the median algorithm represented as $\operatorname{med}\left(\tilde{\sigma}_{g s}^{2}(j)\right)$ to estimate the value of the central sample in a filter window if the sample is detected as noisy. It is possible to use other estimation algorithm such as the FIR median hybrid filter that retains more information about the image.

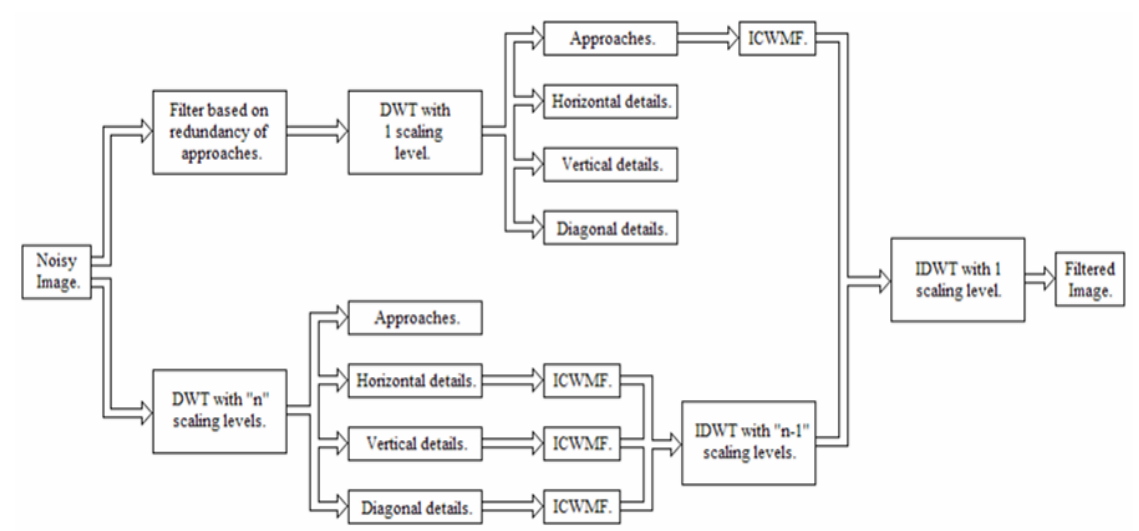

Fig. 1. Block diagram of the proposed filtering scheme of the wavelet domain order statistics filter.

\subsection{Wavelet domain signal dependent rank-ordered mean filter}

Consider a $3 \times 3$ filter window size with a central sample $\tilde{\sigma}_{g s}^{2}(m)=\tilde{\sigma}_{g s}^{2}(k)$, where $m=\left[m_{1}, m_{2}\right]$ is the location of the sample in the image. We define the vector $\varphi(m)$ which contains the neighbor pixels of $\tilde{\sigma}_{g s}^{2}(m)$ into the window as [1]:

$$
\begin{aligned}
\varphi(m)=[ & \widetilde{\sigma}_{g s}^{2}\left(m_{1}-1, m_{2}-1\right), \widetilde{\sigma}_{g s}^{2}\left(m_{1}-1, m_{2}\right), \widetilde{\sigma}_{g s}^{2}\left(m_{1}-1, m_{2}+1\right), \widetilde{\sigma}_{g s}^{2}\left(m_{1}, m_{2}-1\right), \\
& \left.\widetilde{\sigma}_{g s}^{2}\left(m_{1}, m_{2}+1\right), \widetilde{\sigma}_{g s}^{2}\left(m_{1}+1, m_{2}-1\right), \widetilde{\sigma}_{g s}^{2}\left(m_{1}+1, m_{2}\right), \widetilde{\sigma}_{g s}^{2}\left(m_{1}+1, m_{2}+1\right)\right]
\end{aligned}
$$

The samples of the previous vector can be ordered to obtain the vector $v(m)=\left[v_{1}(m), v_{2}(m), \ldots, v_{8}(m)\right]$, where $v_{1}(m), v_{2}(m), \ldots, v_{8}(m)$ are the rank ordered elements of $\varphi(m)$ that satisfy the condition $v_{1}(m) \leq v_{2}(m) \leq, 5, \leq v_{8}(m)$. Then, the rank ordered mean is defined as [1]:

$$
\operatorname{rom}(m)=\left(v_{4}(m)+v_{5}(m)\right) / 2
$$

The vector that contains the rank ordered differences $\operatorname{rod}(m)$ is [1]

$$
\operatorname{rod}(m)=\left[\operatorname{rod}_{1}(m), \operatorname{rod}_{2}(m), \operatorname{rod}_{3}(m), \operatorname{rod}_{4}(m)\right]
$$


where $\operatorname{rod}_{i}(m)=\left\{\begin{array}{l}v_{i}(m)-\tilde{\sigma}_{g s}^{2}(m) \quad \text { for } \tilde{\sigma}_{g s}^{2}(n) \leq \operatorname{rom}(m) \\ \tilde{\sigma}_{g s}^{2}(m)-v_{9-i}(m) \text { otherwise }\end{array}\right.$, and $i=1, \ldots, 4$.

Finally, the SD-ROM algorithm detects $\tilde{\sigma}_{g s}^{2}(m)$ as a noisy sample if any of the following conditions is true [1]

$$
\operatorname{rod}_{i}(m)>U_{i}
$$

where $i=1, \ldots, 4, U_{1}, U_{2}, U_{3}$ y $U_{4}$ are thresholds, $U_{1}<U_{2}<U_{3}<U_{4}$, and $U_{1}=8, U_{2}=2 Q U_{3}=40$ $U_{4}=50[1]$.

\subsection{Wavelet domain adaptive center weighed median filter}

The adaptive center weighed median (ACWM) filter uses an adaptive operator which forms estimators based on the differences between the central sample of the filter window and the values from the CWM Filter with variations of its central weight. The ACWM filter is presented as [9]

$$
\theta_{A C W M}= \begin{cases}\theta_{C W M}^{1}, & d_{k}>U_{k}, \\ \tilde{\sigma}_{g s}^{2}, & \text { otherwise, }\end{cases}
$$

where $d_{k}=\left|\theta_{C W M}^{m}-\tilde{\sigma}_{g s}^{2}\right|$ are the differences between the variable CWM Filter and the central sample in the filtering window, $\theta_{C W M}^{m}=\operatorname{med}\left\{\left[\tilde{\sigma}_{g s}^{2}\right], m \diamond \tilde{\sigma}_{g s}^{2}\right\}$ is the variable CWM filter with its weight $m$. $\left[\tilde{\sigma}_{g s}^{2}\right]_{i}$ are the samples contained in the filter window, $U_{k}=s \cdot M A D+\vartheta_{k}$ are the filter thresholds and $M A D=\operatorname{med}\left\{\left[\tilde{\sigma}_{g s}^{2}\right]_{i}-\theta_{C W M}^{1} \mid\right\}$ is the median absolute deviations from the median. The parameter $s$ gives necessary robustness to the filter and varies between $0 \leq s \leq 0.6$. The optimal values for these parameters are $\vartheta_{k}=\left[\vartheta_{0}, \vartheta_{1}, \vartheta_{2}, \vartheta_{3}\right]=[40,25,10,5]$ and $s=0.3[9]$.

\subsection{Wavelet domain median M-type K-nearest neighbor filter}

The algorithm of median M-type KNN (MMKNN) filter can be written as [10]

$$
\hat{e}_{M M K N N}^{(q)}(i, j)=\operatorname{med}\left\{g^{(q)}(i+m, j+n)\right\}
$$

where $g^{(q)}(i+m, j+n)$ it is a set of $K_{c}$ samples with weight according to the function $\psi\left(\tilde{\sigma}_{g s}^{2}\right)$ required to carry out a comparison with the estimation of the previous step $\hat{e}_{M M K N N}^{(q-1)}(i, j)$. The initial estimator is $\hat{e}_{M M K N N}^{(0)}(i, j)=\tilde{\sigma}_{g s}^{2}(i, j), \tilde{\sigma}_{g s}^{2}(i, j)$ is the central value of the filtering window, and $\hat{e}_{M M K N N}^{(q)}(i, j)$ is the estimator from iteration $q$.

The parameter $K_{c}$ reflects the local data activity and the impulsive presence [10]

$$
K_{c}(i, j)=\left[K_{\min }+a S\left(\tilde{\sigma}_{g s}^{2}(i, j)\right)\right] \leq K_{\max }
$$


where $a$ controls the detail detection, $K_{\min }$ is the minimum number of nearest neighbors to remove the noise, and $K_{\max }$ is the maximum number of neighbors used for the detection of edges and fine details. The optimal values for these parameters are $a=2$ and $K_{\min }=5$, and $S\left(\tilde{\sigma}_{g s}^{2}(i, j)\right)$ is the impulsive detector defined as [10]

$$
S\left(\widetilde{\sigma}_{g s}^{2}(i, j)\right)=\frac{\operatorname{med}\left\{\left|\tilde{\sigma}_{g s}^{2}(i, j)-\tilde{\sigma}_{g s}^{2}(i+m, j+n)\right|\right\}}{\operatorname{MAD}\left\{\tilde{\sigma}_{g s}^{2}(i, j)\right\}}+0.5 \frac{\operatorname{MAD}\left\{\tilde{\sigma}_{g s}^{2}(i, j)\right\}}{\operatorname{med}\left\{\tilde{\sigma}_{g s}^{2}(i+k, j+l)\right\}}
$$

where $\left.M A D_{n}=\operatorname{med}\left\{\left(\tilde{\sigma}_{g s}^{2}\right)_{i}-M_{n}\right\}\right\}$ is the median of absolute deviations from median, $M_{n}=\operatorname{med}\left\{\left(\tilde{\sigma}_{g s}^{2}\right)_{i}\right\},\left(\tilde{\sigma}_{g s}^{2}\right)_{i}$ is the sample of the window that goes from left to right and up to down, and $M_{n}$ is the median of samples in the filtering window.

We also use the simple influence function in the MMKNN filter [10],

$$
\psi_{\text {cut }(r)}\left(\widetilde{\sigma}_{g s}^{2}\right)= \begin{cases}\tilde{\sigma}_{g s}^{2}, & \left|\tilde{\sigma}_{g s}^{2}\right| \leq r, \\ 0, & \text { otherwise }\end{cases}
$$

where $r$ is a parameter between 0 to 255 .

\subsection{Wavelet domain FIR median hybrid filter}

There exist different types of these filters which offer the possibility of choosing the number of sub-filters, their type, as well as the weights and the type of window. In this case, a FIR median hybrid (FIRMH) filter is defined by means of the use of filter windows shown in Fig. 3 and Eq. (2).

$$
\begin{aligned}
& F_{1}=\operatorname{med}\left\{Y_{N}, Y_{E}, Y_{S}, Y_{O}, \widetilde{\sigma}_{g s_{m+1, m+1}}^{2}\right\}=m e d\left\{\begin{array}{l}
\frac{1}{m} \sum_{i=1}^{m} \widetilde{\sigma}_{g s_{i, m+1}}^{2}, \frac{1}{m} \sum_{i=m+2}^{z} \tilde{\sigma}_{g s_{m+1, i}}^{2}, \\
\frac{1}{m} \sum_{i=m+2}^{2} \tilde{\sigma}_{g s_{i, m+1}}^{2}, \frac{1}{m} \sum_{i=1}^{m} \tilde{\sigma}_{g s_{m+1, i}}^{2}, \widetilde{\sigma}_{g s_{m+1, m+1}}^{2}
\end{array}\right\}, \\
& F_{2}=\operatorname{med}\left\{Y_{N O}, Y_{S O}, Y_{S E}, Y_{N E}, \widetilde{\sigma}_{g s_{m+1, m+1}}^{2}\right\}=\operatorname{med}\left\{\begin{array}{l}
\frac{1}{m} \sum_{i=1}^{m} \widetilde{\sigma}_{g s_{i, i}}^{2}, \frac{1}{m} \sum_{i=m+2}^{m} \widetilde{\sigma}_{g s_{z-i+1, i}}^{2}, \\
\frac{1}{m} \sum_{i=m+2}^{z} \widetilde{\sigma}_{g s_{i, i}}^{2}, \frac{1}{m} \sum_{i=m+2}^{z} \widetilde{\sigma}_{g s_{z-i+1, i}}^{2}, \widetilde{\sigma}_{g s_{m+1, m+1}}^{2}
\end{array}\right\}, \\
& F_{3}=\operatorname{med}\left\{F_{1}, F_{2}, \tilde{\sigma}_{g s_{m+1, m+1}}^{2}\right\},
\end{aligned}
$$

where $z$ is the measurement in pixels of a side of the used square window in the analysis and $m$ is obtained from $m=(z-1) / 2$, the $Y$ are simply average filters, $F_{1}$ and $F_{2}$ are median filters and $F_{3}$ is the result of a FIR median hybrid filter. 

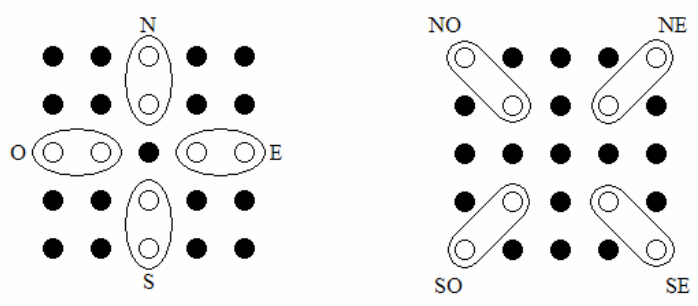

Fig. 3. Filter windows for the FIR Median Hybrid Filter.

\section{Experimental Results}

Through simulation we assessed the properties of the proposed filters via the crossed correlation index (CCI), peak signal noise relation (PSNR), mean square error (MSE), mean absolute error (MAE), mean chromaticity error (MCRE), and normalized color difference (NCD) criteria [1-3].

In the simulation results that we present here, the color image "Lena" was degraded with $20 \%$ of impulsive noise and 0.2 of variance of speckle noise, the wavelet used for the analysis is the dB5 with five levels of decomposition, and a 3x3 filtering window in most of the cases.

The proposed wavelet redundancy of approaches filter (WRAF), wavelet iterative center weighted median using redundancy of approaches filter (WICWMRAF), wavelet signal dependent rank-ordered mean filter (WSDROMF), wavelet adaptive center weighted median filter (WACWMF), wavelet median M-type K-nearest neighbor filter (WMMKNNF), and wavelet FIR median hybrid filter (WFIRMHF) were compared with the wavelet iterative median filter (WIMF) [7] and wavelet iterative center weighted median filter (WICWMF) [7] in terms of CCI, PSNR, MSE, MAE, MCRE and NCD to demonstrate the good quality of color imaging of the proposed filters in both objective and subjective senses.

\subsection{Impulsive noise suppression}

In the sense of impulsive noise suppression, we present in Table 1, the performance results obtained by use of the WMMKNNF in the case of different decomposition levels $(1, \ldots, 5)$ in the image "Lena" degraded with $20 \%$ of impulsive noise to demonstrate how to improve the performance results by the use of five decomposition levels into the proposed filters. Fig. 4 presents the visual results to apply the WMMKNNF with 5 decomposition levels in the restored zoom part of the image "Mandrill" degraded with $20 \%$ of impulsive noise. From this figure we observe that the restored image appears to have a good subjective quality.

Table 2 shows the performance results using the same image by means of use of different filters. From the results presented in this table we observe that the proposed 


\section{Wavelet Order Statistics}

filters provide the best results in the objective criteria in comparison with the traditional filters in the wavelet domain.

Table 1. Performance results in the case of impulsive noise in the image "Lena" produced by WMMKNNF filter.

\begin{tabular}{|c|c|c|c|c|c|c|c|}
\hline \multicolumn{8}{|c|}{ “Lena” Image degraded with $20 \%$ of impulsive noise } \\
\hline Scale & CCI & $\begin{array}{c}\text { PSNR } \\
\text { red }\end{array}$ & $\begin{array}{l}\text { PSNR } \\
\text { green }\end{array}$ & $\begin{array}{c}\text { PSNR } \\
\text { blue }\end{array}$ & $\begin{array}{c}\text { MSE } \\
\text { red }\end{array}$ & $\begin{array}{l}\text { MSE } \\
\text { green }\end{array}$ & $\begin{array}{l}\text { MSE } \\
\text { blue }\end{array}$ \\
\hline 1 & 0.9259 & 29.7595 & 31.1900 & 31.6032 & 3316.200 & 2874.165 & 2757.850 \\
\hline 2 & 0.9713 & 39.4678 & 41.5402 & 42.2203 & 1256.071 & 1020.963 & 953.8331 \\
\hline 3 & 0.9844 & 45.1818 & 48.0759 & 49.0576 & 709.3485 & 531.0914 & 481.4325 \\
\hline 4 & 0.9877 & 47.3538 & 50.6314 & 51.8953 & 570.8607 & 411.3233 & 362.4883 \\
\hline 5 & 0.9886 & 47.9475 & 51.4005 & 52.7380 & 537.9561 & 380.8749 & 333.1943 \\
\hline \multicolumn{8}{|c|}{ “Lena” Image degraded with $20 \%$ of impulsive noise } \\
\hline Scale & $\begin{array}{c}\text { MAE } \\
\text { Red }\end{array}$ & $\begin{array}{l}\text { MAE } \\
\text { Green }\end{array}$ & $\begin{array}{l}\text { MAE } \\
\text { Blue }\end{array}$ & $\begin{array}{c}\text { MCRE } \\
\text { Red }\end{array}$ & $\begin{array}{c}\text { MCRE } \\
\text { green }\end{array}$ & $\begin{array}{c}\text { MCRE } \\
\text { blue }\end{array}$ & NCD \\
\hline 1 & 34.9411 & 34.1184 & 34.3089 & 0.0509 & 0.0442 & 0.0424 & 0.4802 \\
\hline 2 & 26.4170 & 24.1706 & 23.6035 & 0.0193 & 0.0157 & 0.0146 & 0.3130 \\
\hline 3 & 20.7359 & 17.8541 & 17.0267 & 0.0109 & 0.0081 & 0.0074 & 0.2276 \\
\hline 4 & 18.7769 & 15.7317 & 14.8150 & 0.0087 & 0.0063 & 0.0055 & 0.1989 \\
\hline 5 & 18.2160 & 15.0775 & 14.1699 & 0.0082 & 0.0058 & 0.0051 & 0.1911 \\
\hline
\end{tabular}

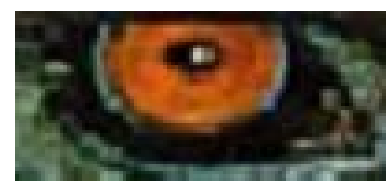

a)

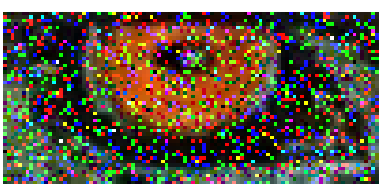

b)

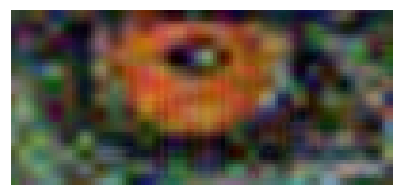

c)

Fig. 4. Subjective visual quantities of restored zoom part of the color image "Mandrill”, a) original image, b) input noisy image corrupted by $20 \%$ impulsive noise in each a channel, c) WMMKNNF filtered image.

Table 2. Performance results in the case of $20 \%$ of impulsive noise in the image "Lena".

\begin{tabular}{cccccc}
\hline Filters & CCI & PSNR & MAE & MCRE & NCD \\
\hline WIMF & 0.9715 & 40.6734 & 24.7969 & 0.0172 & 0.3294 \\
WICWMF & 0.9715 & 40.6734 & 24.7969 & 0.0172 & 0.3294 \\
WRAF & 0.9805 & 44.4936 & 20.8426 & 0.0117 & 0.2718 \\
WICWMRAF & 0.9886 & 50.6952 & 15.8213 & 0.0063 & 0.1911 \\
WSDROMF & 0.9886 & 50.6952 & 15.8213 & 0.0063 & 0.1911 \\
WACWMF & 0.9886 & 50.6952 & 15.8213 & 0.0063 & 0.1911 \\
WMMKNNF & 0.9886 & 50.6953 & 15.8211 & 0.0063 & 0.1911 \\
WFIRMHF & 0.9886 & 50.6992 & 15.8189 & 0.0063 & 0.1911 \\
\hline
\end{tabular}




\subsection{Speckle noise suppression}

Table 3 presents the performance results by means of use different filters in the image "Lena" degraded with 0.2 of variance of speckle noise. Fig. 5 shows the subjective visual quantities of a restored zoom part of the color image "Lena". From these results we observe that the proposed filters provide better speckle noise suppression, detail preservation, and color retention in comparison with the reference filters.

Table 3. Performance results in the case of speckle noise in the image "Lena" produced by different filters.

\begin{tabular}{cccccc}
\hline Filters & CCI & PSNR & MAE & MCRE & NCD \\
\hline WIMF & 0.9773 & 43.7164 & 22.2675 & 0.0138 & 0.2617 \\
WICWMF & 0.9773 & 43.7164 & 22.2675 & 0.0138 & 0.2617 \\
WRAF & 0.9853 & 48.0338 & 18.2333 & 0.0093 & 0.2062 \\
WICWMRAF & 0.9909 & 53.8602 & 14.1506 & 0.0056 & 0.1489 \\
WSDROMF & 0.9909 & 53.8602 & 14.1506 & 0.0056 & 0.1489 \\
WACWMF & 0.9909 & 53.8602 & 14.1507 & 0.0056 & 0.1489 \\
WMMKNNF & 0.9909 & 53.8603 & 14.1506 & 0.0056 & 0.1489 \\
WFIRMHF & 0.9909 & 53.8608 & 14.1509 & 0.0056 & 0.1489 \\
\hline
\end{tabular}

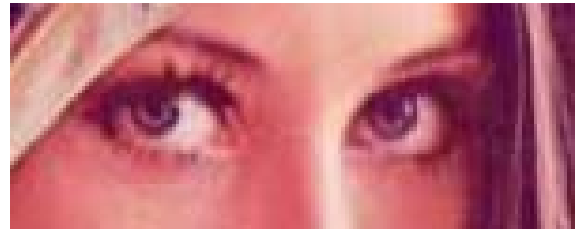

a)

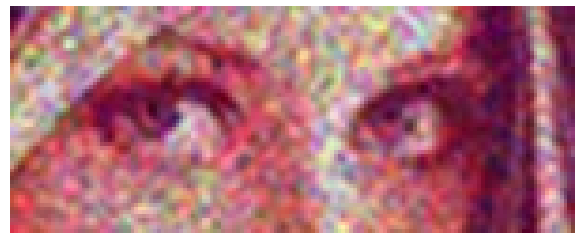

c)

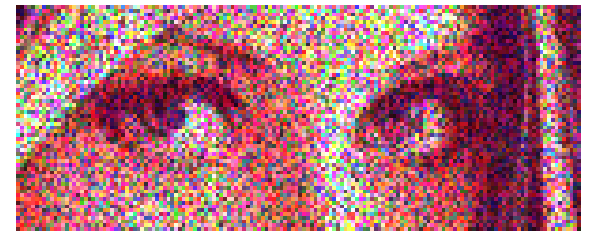

b)

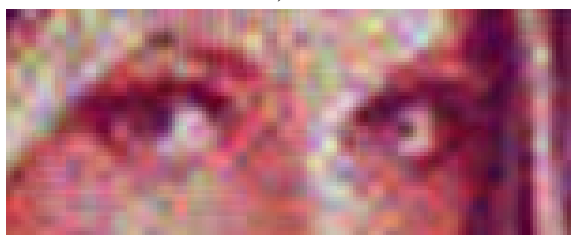

d)

Fig. 5. Subjective visual quantities of restored zoom part of color image "Lena”. a) Original image, b) input noisy image corrupted by 0.2 of variance of speckle noise in each a channel, c) WRAF filtered image, and d) WACWMF filtered image.

\subsection{Mixture of impulsive and speckle noise suppression}

We also applied the proposed filters to suppress mixtures of impulsive and speckle noise. Table 4 depicts the performance results in the image "Lena" degraded with $20 \%$ of 
impulsive noise mixed with 0.2 of variance of speckle noise by use of different filters. In Fig. 6 we present the subjective results to apply different filters in a zoom part of image "Lena”. We observe that from the results obtained in Table 4 and Fig. 6, the proposed filters show a better performance in comparison with the filters used for comparison.

Table 4. Performance results in the case of mixture of $20 \%$ of impulsive noise and 0.2 of variance of speckle noise.

\begin{tabular}{cccccc}
\hline Filters & CCI & PSNR & MAE & MCRE & NCD \\
\hline WIMF & 0.9535 & 36.5095 & 32.3755 & 0.0281 & 0.3958 \\
WICWMF & 0.9535 & 36.5095 & 32.3755 & 0.0281 & 0.3958 \\
WRAF & 0.9691 & 40.4136 & 27.2201 & 0.0199 & 0.3194 \\
WICWMRAF & 0.9812 & 46.3350 & 21.6653 & 0.0127 & 0.2370 \\
WSDROMF & 0.9812 & 46.3345 & 21.6659 & 0.0127 & 0.2370 \\
WACWMF & 0.9812 & 46.3345 & 21.6659 & 0.0127 & 0.2370 \\
WMMKNNF & 0.9812 & 46.3351 & 21.6651 & 0.0127 & 0.2370 \\
WFIRMHF & 0.9813 & 46.3374 & 21.6646 & 0.0127 & 0.2370 \\
\hline
\end{tabular}

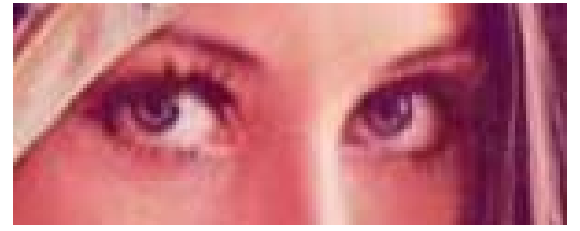

a)

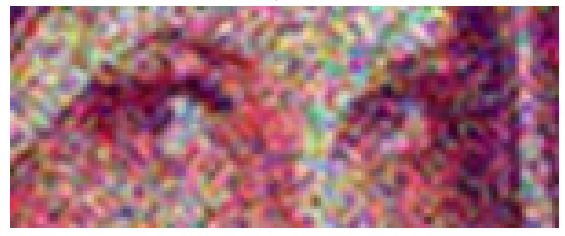

c)

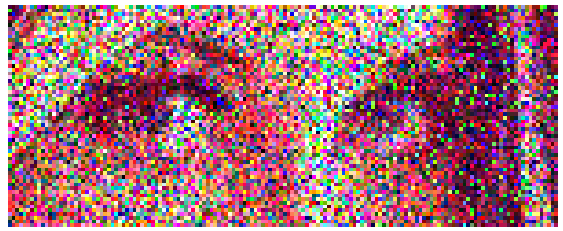

b)

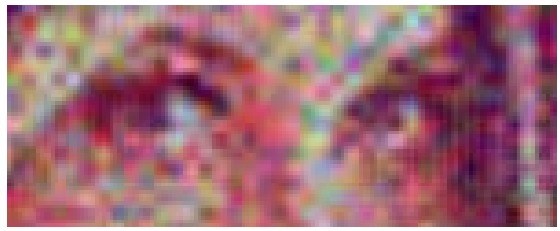

d)

Fig. 6. Subjective visual quantities of restored zoom part of color image "Lena". a) Original image, b) input noisy image corrupted by $20 \%$ of impulsive noise mixed with 0.2 of variance of speckle noise in each a channel, and c) WRAF filtered image; d) WMMKNNF filtered image.

\section{Conclusions}

We present different implementations of order statistics filters in wavelet domain. The proposed filters constitute a good tool to deal with impulsive and speckle noise filtering in color image applications. It is obviously true that the use of different order statistics filters into the two proposed blocks detection in the wavelet domain can increase the processing time. 


\section{Acknowledgements}

The authors thank the National Polytechnic Institute of Mexico for its support.

\section{References}

1. S. K. Mitra and G. L. Sicuranza, Nonlinear Image Processing (Academic Press, San Diego, CA, 2001).

2. J. Astola and P. Kuosmanen, Fundamentals of Nonlinear Digital Filtering (CRC Press, Boca Raton-New York, 1997).

3. K. N. Plataniotis and A. N. Venetsanopoulos, Digital Signal Processing/Color Image Processing and Applications (Springer-Verlag Berlin Heidelberg, 2000).

4. Z. Cai, T. H. Cheng, C. Lu, and K. R. Subramanium, Efficient wavelet-based image denoising algorithm, Electron. Lett. 37 (11) 683 (2001). doi:10.1049/el:20010466

5. S. G. Chang, B. Yu, and M. Vettereli, Adaptive wavelet thresholding for image denoising and compression, IEEE Trans. Image Process. 9 (9) 1532 (2000). doi:10.1109/83.862633

6. J. Martínez Valdés, F. J. Gallegos Funes, and M. A. Acevedo Mosqueda, Wavelet Domain Statistical Order Filter using the Tri-State Median Filter algorithm, Proc. 2nd Int. Conf. Electrical and Electronics Engineering (IEEE, Mexico, 2005) pp. 32-35.

7. S. M. Mahbubur Rahman and Md. Kamrul Hasan, Wavelet-domain iterative center weighted median filter for image denoising, Signal Processing 83, 1001 (2003). doi:10.1016/S0165-1684(02)00502-9

8. T. Chen, K. Ma, and L. Chen, Tri-State Median Filter for image denoising, IEEE Trans. Image Process. 8 (12), 1834 (1999). doi:10.1109/83.806630

9. T. Chen and H. R. Wu, Adaptive impulse detection using center-weighted median filters, IEEE Signal Processing Letters 8 (1), 1 (2001). doi:10.1109/97.889633

10. F. Gallegos and V. Ponomaryov, Real-time image filtering scheme based on robust estimators in presence of impulsive noise, Real Time Imaging 8 (2), 78 (2004). 